\title{
Resíduos agroindustriais como substrato para a produção de lipases microbiana:
}

\section{uma revisão}

\author{
Agro-industrial waste as a substrate for the production of microbial lipases: a review \\ Residuos agroindustriales como sustrato para la producción de lipasas microbianas: una revisión
}

Recebido: 01/02/2021 | Revisado: 05/02/2021 | Aceito: 09/02/2021 | Publicado: 16/02/2021

Eduardo Henrique Santos Guedes ORCID: https://orcid.org/0000-0003-3189-9576

Universidade Federal do Tocantins, Brasil E-mail: edu3-d@hotmail.com

André Leonardo dos Santos

ORCID: https://orcid.org/0000-0001-7795-2825 Universidade Federal Rural do Rio de Janeiro, Brasil E-mail: anddreleo@gmail.com

Andréia Ibiapina

ORCID: https://orcid.org/0000-0003-2523-9555

Universidade Federal do Tocantins, Brasil

E-mail: ibiapinaandreia@gmail.com

Aynaran Oliveira Aguiar

ORCID: https://orcid.org/0000-0002-3596-0699

Universidade Federal do Tocantins, Brasil

E-mail: aynaranaguiar@gmail.com

Camila Mariane da Silva Soares

ORCID: https://orcid.org/0000-0002-6194-5126

Universidade Federal de Lavras, Brasil

E-mail: cammisoares06@gmail.com

Patrícia Oliveira Vellano

ORCID: https://orcid.org/0000-0001-9956-054X

Universidade do Norte do Paraná, Brasil

E-mail: patfarma25@hotmail.com

Lucas Samuel Soares dos Santos

ORCID: https://orcid.org/0000-0003-2522-0176

Universidade Federal do Tocantins, Brasil

E-mail: 1santos@mail.uft.edu.br

Aloísio Freitas Chagas Junior

ORCID: https://orcid.org/0000-0002-7489-8701

Universidade Federal do Tocantins, Brasil

E-mail: chagasjraf@uft.edu.br

\begin{abstract}
Resumo
O objetivo desse trabalho foi realizar uma pesquisa bibliográfica sobre a utilização de resíduos agroindústrias como substrato para produção de lipase microbiana, identificando os principais microrganismos, tempo de fermentação e a inclusão de indutores ao meio. Trinta trabalhos foram selecionados e formaram um banco de dados. Pesquisas no Brasil representaram $60 \%$ das publicações utilizadas, o resíduo (torta e borra) de dendê e soja foram os mais relatados representando 13,3\%, os fungos filamentosos foram os mais estudados, o gênero Aspergillus foi o mais encontrado totalizando $36,6 \%$ e em especial a espécie $A$. niger, que foi estudada por oito pesquisadores. Em relação ao tempo, as fermentações variaram de 20 a $336 \mathrm{~h}$, sendo que o tempo de $48 \mathrm{~h}$ foi utilizado por sete pesquisadores. Dentre as 30 publicações foi possível verificar que em 13 (43\%) delas os autores não utilizaram nenhum tipo de indutor para suplementar o resíduo agroindustrial, porém a utilização de fontes lipídicas e principalmente de nitrogênio aumentaram significativamente a produção de lipase. A maioria das pesquisas foram conduzidas em FES (73,3\%). Utilizar resíduos agroindustriais e suplementar o meio com indutores com preços mais baixos, além de potencializar a produção pode tornar o produto final mais barato.
\end{abstract}

Palavras-chave: Lipase; Microrganismos; Fermentação; Resíduo agroindustrial.

\begin{abstract}
The objective of this work was to carry out a bibliographic research on the use of agro-industrial waste as a substrate for the production of microbial lipase, identifying the main microorganisms, fermentation time and the inclusion of inducers in the medium. Thirty papers were selected and formed a database. Research in Brazil accounted for $60 \%$ of the publications used, the palm oil and soy residue (cake and sludge) were the most reported representing $13.3 \%$, the
\end{abstract}


filamentous fungi were the most studied, the Aspergillus genus was the most found totaling $36.6 \%$ and in particular the species A. niger, which was studied by eight researchers. Regarding the time, fermentations ranged from 20 to 336 $\mathrm{h}$, and the time of $48 \mathrm{~h}$ was used by seven researchers. Among the 30 publications it was possible to verify that in 13 $(43 \%)$ of them the authors did not use any type of inducer to supplement the agro-industrial residue, however the use of lipid sources and mainly of nitrogen significantly increased the production of lipase. Most surveys were conducted in FES (73.3\%). Using agro-industrial waste and supplementing the environment with lower priced inductors, in addition to boosting production can make the final product cheaper.

Keywords: Lipase; Microorganisms; Fermentation; Agro-industrial waste.

\section{Resumen}

El objetivo de este trabajo fue realizar una investigación bibliográfica sobre el uso de residuos agroindustriales como sustrato para la producción de lipasa microbiana, identificando los principales microorganismos, el tiempo de fermentación y la inclusión de inductores en el medio. Se seleccionaron treinta artículos y se formó una base de datos. La investigación en Brasil representó el 60\% de las publicaciones utilizadas, el aceite de palma y los residuos de soja (tortas y lodos) fueron los más reportados con un 13,3\%, los hongos filamentosos fueron los más estudiados, el género Aspergillus fue el más encontrado, totalizando un $36,6 \%$. y en particular la especie A. niger, que fue estudiada por ocho investigadores. En cuanto al tiempo, las fermentaciones variaron de 20 a $336 \mathrm{~h}$, siendo el tiempo de $48 \mathrm{~h}$ utilizado por siete investigadores. Entre las 30 publicaciones se pudo comprobar que en $13(43 \%)$ de ellas los autores no utilizaron ningún tipo de inductor para suplementar el residuo agroindustrial, sin embargo el uso de fuentes lipídicas y principalmente de nitrógeno incrementó significativamente la producción de lipasa. La mayoría de las encuestas se realizaron en FES (73,3\%). Usar residuos agroindustriales y complementar el medio ambiente con inductores de menor precio, además de impulsar la producción, puede abaratar el producto final.

Palabras clave: Lipasa; Microorganismos; Fermentación; Residuos agroindustriales.

\section{Introdução}

A agroindústria e a indústria de alimentos são as maiores produtoras de resíduos líquidos e sólidos, com isso esses dejetos representam um grande problema quanto ao tratamento final e potencial poluente. Porém a conscientização ecológica vem mudando ao longo dos tempos e, ao contrário do que acontecia no passado, os resíduos agroindustriais passaram a ser considerados subprodutos de alto valor agregado, não sendo mais destinado aos aterros sanitários, ração animal ou adubo, pois passaram a ser vistos como subprodutos ricos em biomassa com nutrientes de alto valor (Pinto et al., 2005).

Utilizando técnicas biotecnológicas, os resíduos agroindustriais podem se converter, através de microrganismos, em bioprodutos de grande interesse industrial, como as enzimas através das fermentações em estado sólido ou fermentações submersas (Farias et al., 2014).

Atualmente a fermentação em estado sólido (FES) vem sendo a mais utilizada, pois muitos subprodutos agroindustriais podem ser aproveitados como substratos para a produção de enzimas, tal fato está relacionado principalmente por colaborar para a redução do custo operacional da produção enzimática e dispor de atributos físicos e químicos que beneficiam o crescimento microbiano (Ferreira et al., 2011).

Segundo Treichel et al. (2010), utilizar resíduos agroindustriais fornece opções em substratos e podem auxiliar a resolver problemas de poluição, que caso contrário, pode ser causado pela sua eliminação, dessa forma é essencial a escolha de um bom resíduo pois seu potencial é um dos fatores mais importantes no processo fermentativo, além do mais a escolha está relacionada ao custo e a sua disponibilidade e ainda à natureza do composto para produzir a enzima de interesse.

Por possuírem açúcares, fibras, materiais hidrolisáveis e fermentáveis, houve um interesse em valorizar dos resíduos e assim novas olhares foram conferidos a folhas, caules, sementes, palhas, bagaços, cascas, farelos, polpas, águas residuais e outros, tornando-se subprodutos com valor agregado e em substituição de substratos comumente utilizados (Takeyama et al., 2020).

Os microrganismos, como bactérias, leveduras e bolores, são reconhecidos como potenciais em produção de lipases extracelulares (Treichel et al., 2010). Os principais gêneros produtores de lipases são fungos filamentosos, especialmente do 
gênero Aspergillus (Penha et al., 2016), as bactérias dos gêneros Bacillus, Pseudomonas, Burkholderia e Staphylococcus e leveduras como a Candida, Yarrowia, Pichia, Rhodotorula e Saccharomycopsis (Liu \& Kokare, 2017).

As lipases são enzimas hidrolíticas que catalisam a hidrólise de triacilglicerol em glicerol e ácidos graxos livres (Gupta et al., 2004). Por isso são empregadas em setores industriais distintos, como na produção de surfactantes, detergentes e tratamento de resíduos ricos em óleos e gorduras na indústria química, nas ciências da saúde compõe medicamentos, em diagnósticos, cosméticos ou antibióticos e bastante utilizada no setor alimentício na formulação de emulsificantes, conversão de lipídios, elaboração de margarinas e na produção de aromas e maturação de queijos e embutidos cárneos, no setor ambiental para a produção de biodiesel (Colla, Reinehr e Costa, 2013).

Vários autores vêm propondo a utilização de resíduos agroindustriais como resíduos popularmente chamado de torta de dendê (Silveira et al., 2015), farelo de arroz (Putri et al., 2020), farelo de trigo (Dobrev et al., 2018) torta do óleo de canola (Rehman et al., 2019) dentre outros resíduos como indutores para a produção de enzimas.

Deste modo o objetivo desse estudo é reunir e fornecer informações acerca de publicações sobre a utilização de resíduas agroindústrias que foram utilizados como substrato para produção de lipase, identificando os principais resíduos, microrganismos, tempo de fermentação e a utilização de indutores suplementares por meio de uma revisão crítica da literatura, como também identificar aspectos pouco explorados cientificamente.

\section{Metodologia}

\subsection{Seleções dos trabalhos}

Este estudo trata-se um uma pesquisa quantitativa exploratória de revisão da literatura utilizando a técnica de mineração de dados como procedimento metodológico objetivando um conhecimento de produções científicas (Pereira et al., 2018).

Os bancos de dados são um conjunto digital que contém artigos em periódico, livros, notas técnicas, publicados com resultados das pesquisas de autores. Segundo Ruas (2014) o processo de Extração de Conhecimento em base de dados é conhecido como KDD (Knowledge Discovery in Databases) que visa coletar dados que tenham afinidade de interesse por assunto como também ser valido para cada dado encontrado.

Para a coleta de trabalhos publicados na literatura foram utilizados os principais bancos de dados: Web of Science, Google Acadêmico, Scopus, Scienc Direct, Periódico CAPES, e PubMed. O levantamento foi realizado no período de agosto a setembro de 2020, no qual foram utilizadas palavras-chaves em inglês e português, como "Lipase", "Produção" e "Resíduo Agroindustrial".

\subsection{Refinamento dos dados}

Como critério de seleção foi empregado: I- Data de publicação (2010 a 2020), apenas artigos originais e em idioma inglês; II-Trabalhos que informassem sobre a produção lipolítica sobre fermentação em estado sólido ou submerso em resíduos agroindustriais; e III- Informou-se também o tempo de fermentação e a utilização ou não de substâncias indutoras ou para suplementação do meio. O critério de exclusão aplicou-se aos trabalhos que não abordassem as exigências dos critérios de inclusão.

\subsection{Organização dos dados e realização das análises}

Após a reunião dos artigos foi realizada uma leitura do resumo para selecionar aquele que dispuserem de informações correntes com o intuito do trabalho e posteriormente foram codificados e filtrados. 


\section{Resultados e Discussão}

A pesquisa bibliográfica utilizada neste estudo foi baseada na consulta de trabalhos publicados nos últimos 10 anos de acordo com as palavras-chaves e base de dados.

Mediante a análise dos trabalhos, foram selecionadas obras que apresentaram resultados expressivos, utilizando metodologias de otimização ou não, de valores máximos de atividade lipolítica. Foram selecionadas 30 publicações sobre a utilização de resíduos agroindústrias para a produção de lipase microbiana. A Tabela 1 apresenta as publicações selecionadas e país de origem.

Tabela 1. Publicações científicas selecionadas de acordo com critérios estabelecidos.

\begin{tabular}{|c|c|c|}
\hline Código & Autores / Ano & Pais \\
\hline 1 & AGUIAR et al., (2018) & Brasil \\
\hline 2 & PENHA et al., (2016) & Brasil \\
\hline 3 & OLIVEIRA, (2014) & Brasil \\
\hline 4 & SOUZA et al., (2013) & Brasil \\
\hline 5 & FERRAZ et al., (2020) & Brasil \\
\hline 6 & TOMBINI et al., (2015) & Brasil \\
\hline 7 & PRABANINGTYAS et al., (2018) & Brasil \\
\hline 8 & FERREIRA et al., (2020) & Brasil \\
\hline 9 & EICHLER, (2018) & Brasil \\
\hline 10 & FARIAS et al., (2014) & Brasil \\
\hline 11 & THIRUNAVUKARASU et al., (2016) & Índia \\
\hline 12 & GODOY et al., (2011) & Brasil \\
\hline 13 & PEREIRA et al., (2019) & Brasil \\
\hline 14 & KUMAR et al., (2011) & Índia \\
\hline 15 & REHMAN et al., (2011) & Paquistão \\
\hline 16 & SARAT et al., (2010) & Índia \\
\hline 17 & MOFTAH et al., (2012) & Sérvia \\
\hline 18 & JAIN e NAIK, (2018). & Índia \\
\hline 19 & PUTRI et al, (2020) & Indonésia \\
\hline 20 & COSTA et al., (2017) & Brasil \\
\hline 21 & OLIVEIRA et al., (2018) & Brasil \\
\hline 22 & SUCI et al., (2016) & Indonésia \\
\hline 23 & DOBREV et al., (2018) & Bulgária \\
\hline 24 & REHMAN et al., (2019) & Paquistão \\
\hline 25 & SILVEIRA et al, (2016) & Brasil \\
\hline 26 & FARIAS et al, (2015) & Brasil \\
\hline 27 & AMORIM et al, (2013) & Brasil \\
\hline 28 & JÚNIOR et al., (2016) & Brasil \\
\hline 29 & GARLAPATI e BANERJEE (2010) & Índia \\
\hline 30 & RAJENDRAN e THANGAVELU, (2013). & Índia \\
\hline
\end{tabular}

Fonte: Autores (2021).

Dentre as 30 publicações selecionadas, $60 \%$ foram pesquisas realizadas no Brasil, $20 \%$ na Índia, 6,6\% Paquistão e Indonésia, outros 3,3\% na Bulgária e Sérvia. O Brasil se destaca como o maior gerador dentre as publicações selecionadas pois o crescimento do agronegócio, além de incentivar o desenvolvimento o torna um grande produtor de resíduas agropecuárias e agroindústrias, seja pela perca na produção e também dos materiais gerados na cadeia produtiva, tornaram-se uma fonte importante para a produção de novos materiais (Rosa et al. 2011). 
Pelo cenário prospero vários setores indústrias estão pesquisando novas técnicas para aproveitar e valorizar os resíduos e subprodutos da agroindústria, a tecnologia enzimática vem utilizando esses resíduos para a produção de enzimas de interesse industrial e biotecnológico (Dos Santos et al., 2018). No período pesquisado entre 2010 e 2020, os anos de 2016 e 2018 foram aqueles que apresentaram um maior número de publicações sobre o assunto, seis e oito publicações respectivamente. Rosa et al. (2011), destacam o interesse por fontes renováveis para desenvolver e implementar processos mais sustentáveis e apropriados para transformar biomassa em produtos valiosos, pois é uma necessidade na tentativa de reduzir impactos ambientais, justifica-se assim o aumento nos últimos 10 anos em relação à pesquisa sobre o aproveitamento desses resíduos.

\subsection{Resíduos agroindustriais utilizados como substratos para produção de lipase}

Uma variedade de resíduos foi encontrada nas publicações, sendo o resíduo oriundo da obtenção do azeite de dendê (torta) e farelo de soja os mais relatados dentre as pesquisas ambos representando 13,3\%, entretanto outros resíduos merecem destaques pela sua boa aplicabilidade como substrato como farelo de arroz que representou $10 \%$ na pesquisa, a Figura 1 ilustra a diversidade dos resíduos mais utilizados pelos pesquisadores.

Figura 1. Dimensão dos resíduos agroindustriais mais utilizados como substrato para produção de lipase microbiana.

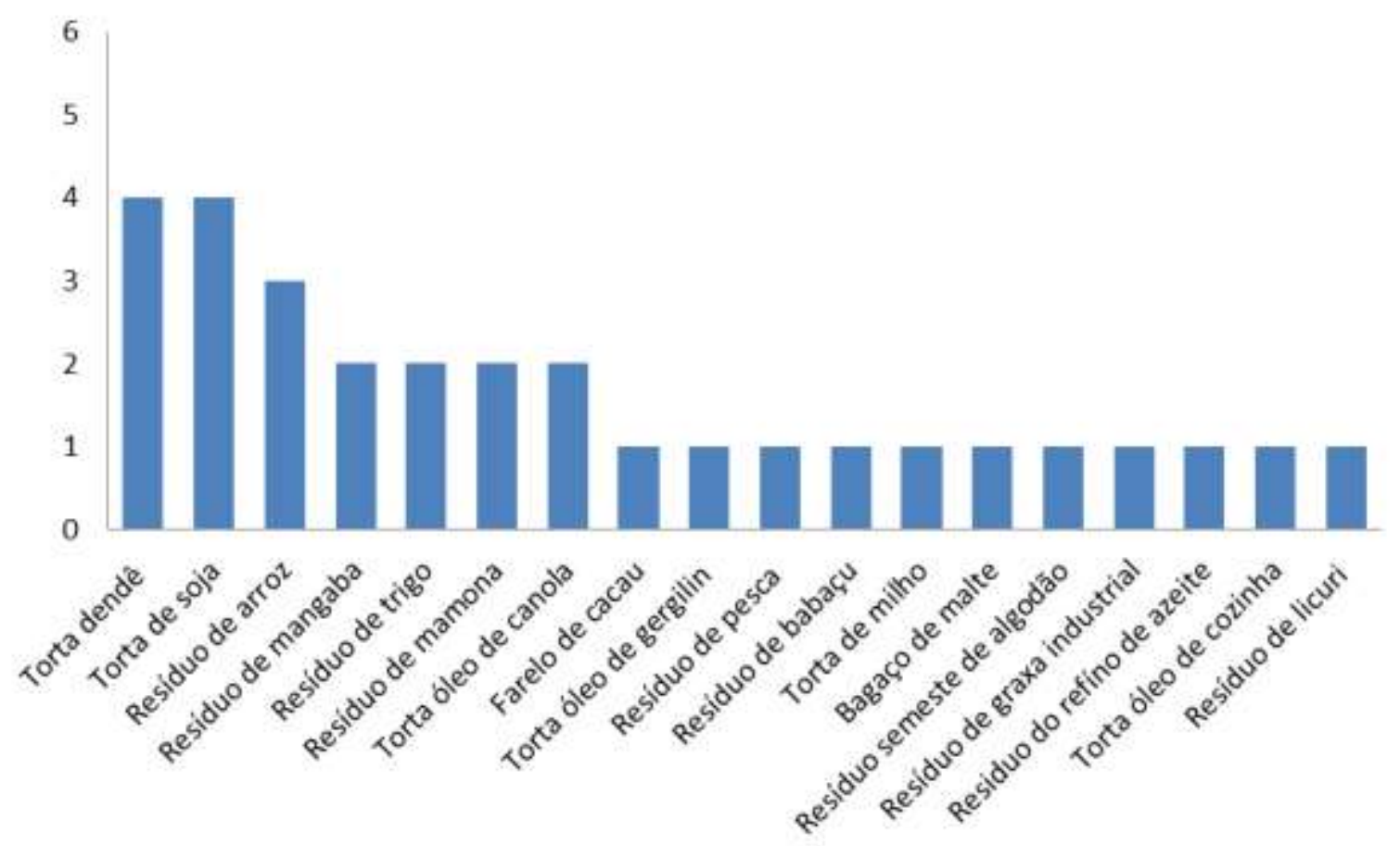

Fonte: Autores (2021).

Os resíduos do dendê são utilizados em sua grande maioria para a alimentação animal principalmente para dietas de peixes em países como a Índia, por apresentarem altos valores de fibra, proteína, óleo residual, carboidratos, cinzas e água, ademais representam um grande problema ambiental (Penha et al., 2016; Sarat et. al., 2010).

Dessa maneira autores como Oliveira et al. (2018), Penha et al. (2016), Silveira et al., (2015) e Sarat et al. (2010), utilizaram os resíduos provenientes da produção do azeite de dendê como substrato principal para a produção de lipases e obtiveram resultados equivalentes a 20,7, 72,5, 15,4 e 6,4 $\mathrm{U} \mathrm{g}^{-1}$ de atividade máxima lipolítica respectivamente. 
Em seguida, os farelos de soja (4 publicações) e arroz ( 3 publicações) foram explorados por outros autores, demostrando assim, que possuem grande potencial na produção de lipase por apresentar altos valores de proteína bruta, lipídeos e carboidratos, autores como Oliveira (2014), Farias et al. (2015), Tombini et al. (2015), Júnior et al. (2016), Costa et al. (2017) e Putri et al. (2020), encontraram bons resultados em relação a atividade máxima lipolítica destes resíduos, que chegaram à $176 \mathrm{U} \mathrm{g}^{-1}$.

Por outro lado além do farelo de soja e arroz, Sarat et. al (2010) elucidam que várias pesquisas nos últimos anos vem utilizando diversos resíduos agrícolas, que são renováveis e abundantemente disponíveis e podem contribuir com agregação de valor, como exemplo a torta do óleo de babaçu e gergelim que têm sido usados como substratos para a produção de lipase, com isso fica evidente a aplicabilidade de diversos tipos de resíduos que podem ser utilizados para produção de lipase corroborando com os resultados do estudo que selecionou 18 tipos diferentes de resíduos.

Salihu et al. (2012) alertam que para a produção de lipase usando resíduos fibrosos requer suplementação de nutrientes ou uma mistura de mais de um substrato ou indutores.

\subsection{Microrganismos produtores de lipase}

Para a produção de lipase a utilização de diversos gêneros microbianos vem aumentando nas últimas décadas devido à grande eficiência que esses organismos possuem em produzir essas moléculas (Martins et al., 2015). As bactérias, fungos filamentosos e leveduras são capazes de produzir lipases extracelular, de modo que os dados mostram que os fungos filamentosos representaram $70 \%$ dos estudos, seguido pelas leveduras $23,3 \%$ e bactérias $6,6 \%$. A Tabela 2 apresenta a proporção das espécies microbianas com resultados de atividade máxima e o resíduo utilizado com o substrato.

Tabela 2. Espécies microbianas produtoras de lipase em diferentes resíduos agroindustriais e seus valores de atividade máxima lipolítica.

\begin{tabular}{|c|c|c|c|}
\hline Espécie & $\begin{array}{c}\text { Atividade máxima de } \\
\text { lipase }\end{array}$ & Resíduo & Referência \\
\hline A. brasiliensis ${ }^{\mathrm{FES}}$ & $8,1 \mathrm{U} \mathrm{g}^{-1}$ & Bagaço de malte & Eichler (2018) \\
\hline A. candidus $^{\mathrm{FES}}$ & $395,1 \mathrm{U} \mathrm{g}^{-1}$ & Farelo de licuri & Farias et al. (2015) \\
\hline A. niger $^{\mathrm{FES}}$ & $20,7 \mathrm{U} \mathrm{g}^{-1}$ & Dendê* & Oliveira et al. (2018) \\
\hline A. niger $^{\mathrm{FES}}$ & $72,5 \mathrm{U} \mathrm{g}^{-1}$ & Torta de dendê & Penha et. al. (2016) \\
\hline A. niger $^{\mathrm{FES}}$ & $244,9 \mathrm{U} \mathrm{g}^{-1}$ & Semente de mangaba* & Souza et al. (2013) \\
\hline A. niger $^{\mathrm{FES}}$ & $163,3 \mathrm{U} \mathrm{g}^{-1}$ & Farelo de soja* & Prabaningtyas et al. (2018) \\
\hline A. niger $^{\mathrm{FES}}$ & $176 \mathrm{U} \mathrm{mL}^{-1}$ & Farelo de arroz & Putri et al. (2020) \\
\hline A. niger $^{\mathrm{FES}}$ & $77 \mathrm{U} \mathrm{g}^{-1}$ & Óleo de palma & Silveira et al. (2016) \\
\hline A. niger $^{\mathrm{FES}}$ & $11,6 \mathrm{U} \mathrm{g}^{-1}$ & Farelo de cacau* & Amorim et al. (2013) \\
\hline A. niger $^{\mathrm{FES}}$ & $19,8 \mathrm{U} \mathrm{g}^{-1}$ & Farelo de arroz & Costa et al. (2017) \\
\hline A. oryzae e japonica ${ }^{\mathrm{FES}}$ & $25 \mathrm{U} \mathrm{g}^{-1}$ & Mamona $^{*}$ & Jain e Naik (2018). \\
\hline Bacillus subtilis ${ }^{\mathrm{FS}}$ & $15,9 \mathrm{U} \mathrm{mL}^{-1}$ & Pesca $*$ & Aguiar et al. (2018) \\
\hline Bacillus subtilis ${ }^{\mathrm{FS}}$ & $4,9 \mathrm{U} \mathrm{mL}^{-1}$ & Óleo de cozinha & Suci et al. (2016) \\
\hline Candida Rugosa ${ }^{\mathrm{FES}}$ & $22,4 \mathrm{U} \mathrm{g}^{-1}$ & Torta de gergelim & $\begin{array}{l}\text { Rajendran e Thangavelu } \\
\text { (2013). }\end{array}$ \\
\hline Candida rugosa ${ }^{\mathrm{FS}}$ & $12,3 \mathrm{U} \mathrm{mL}^{-1}$ & Melaço de soja* & Júnior et al. (2016) \\
\hline
\end{tabular}




\begin{tabular}{|c|c|c|c|}
\hline Candida $_{\text {utilisna }}{ }^{\mathrm{FES}}$ & $25 \mathrm{U} \mathrm{g}^{-1}$ & Torta de azeite & Moftah et al. (2012) \\
\hline Cryptococcus sp. ${ }^{\mathrm{FES}}$ & $753 \mathrm{U} \mathrm{g}^{-1}$ & Semente de algodão* & Thirunavukarasu et al. (2016) \\
\hline G. candidum $^{\mathrm{FES}}$ & $29,4 \mathrm{U} \mathrm{g}^{-1}$ & Torta de milho & Ferreira et al. (2020) \\
\hline Metarhizium anisopliae $^{\mathrm{FES}}$ & $4,4 \mathrm{U} \mathrm{mL}^{-1}$ & Bagaço de babaçu* & Ferraz et al. (2020) \\
\hline P. chrysogenum ${ }^{\mathrm{FES}}$ & $38 \mathrm{U} \mathrm{mL}^{-1}$ & Resíduos de graxa & Kumar et al. (2011) \\
\hline P. crustosum $^{\mathrm{FES}}$ & $136,1 \mathrm{U} \mathrm{g}^{-1}$ & Farelo de arroz & Oliveira (2014) \\
\hline P. notatum ${ }^{\mathrm{FES}}$ & $5335 \mathrm{U} \mathrm{g}^{-1}$ & Torta de canola & Rehman et al. (2011) \\
\hline P. simplicissimum ${ }^{\mathrm{FES}}$ & $155,0 \mathrm{U} \mathrm{g}^{-1}$ & Mamona* & Godoy et al. (2011) \\
\hline Penicillium $s p$ FES & $73,8 \mathrm{U} \mathrm{L}^{-1}$ & Farelo de soja* & Tombini et al. (2015) \\
\hline Pleurotus ostreatus FES & $3256 \mathrm{U} \mathrm{g}^{-1}$ & Torta de canola & Rehman et al. (2019) \\
\hline Rhizopus arrhizus ${ }^{\mathrm{FES}}$ & $1021,8 \mathrm{U} \mathrm{g}^{-1}$ & Farelo de trigo & Dobrev et al. (2018) \\
\hline Rhizopus oryzae $\mathrm{FES}$ & $96.5 \mathrm{U} \mathrm{g}^{-1}$ & Farelo de trigo* & Garlapati e Banerjee (2010) \\
\hline Yarrowia lipolytica FES & $139 \mathrm{U} \mathrm{g}^{-1}$ & Torta de soja ${ }^{*}$ & Farias et al. (2014) \\
\hline Yarrowia lipolytica ${ }^{\mathrm{FS}}$ & $3,5 \mathrm{U} \mathrm{mL}^{-1}$ & Sementes e cascas de manga & Pereira et al. (2019) \\
\hline Yarrowia lipolytica FES & $18,5 \mathrm{U} \mathrm{g}^{-1}$ & Torta de dendê & Sarat et al. (2010) \\
\hline
\end{tabular}

FS: Fermentação Submersa; FES: Fermentação no estado sólido; * Resíduo suplementados com indutores no meio. Fonte: Autores (2021).

Dentre os fungos filamentosos, o Aspergillus foi o género mais encontrado nas publicações totalizando 36,6\% seguido pelo gênero Penicillium (20\%) e em especial a espécie A. niger que foi estudada por 8 pesquisadores. Essa espécie é bastante conhecida e utilizada para a produção de lipase e outros tipos de enzimas extracelulares, comparada às bactérias, por exemplo, possuem propriedades fisiológicas, bioquímicas e enzimáticas que proporcionam suportar e se adaptar em resíduos com baixa umidade crescendo-se facilmente (Dos Santos et al., 2018), tal motivo pode explicar a afinidade dos pesquisadores na escolha de gêneros de fungos filamentosos.

Mesmo sendo o mais estudado dentre os microrganismos o Aspergillus não foi o microrganismos mais eficaz na produção de lipases. Utilizando a torta do óleo de canola Rehman et al. (2011) relatam a produção de $5335 \mathrm{U} \mathrm{g}^{-1}$ por Penicillium notatum. Vale ressaltar que os mesmos autores, em outra publicação no ano de 2019 utilizando o mesmo substrato porém a espécie Pleurotus ostreatus, encontraram valores expressivos de $3256 \mathrm{U} \mathrm{g}^{-1}$, tornando-se assim os maiores valores de produção de lipase encontrada dentro das publicações selecionadas.

Entre as leveduras destaca-se os gêneros Candida e Yarrowia que usualmente são empregadas para produção de lipase, utilizando resíduo da torta de óleo de gergelim Rajendran e Thangavelu (2013) encontraram 22,4 U g-1 utilizando Candida rugosa. Já Farias et al. (2015) encontraram $139 \mathrm{U} \mathrm{g}^{-1}$ por Yarrowia lipolytica em torta de soja como substrato sendo assim o maior valor para as leveduras estudadas nas publicações.

A espécie Bacillus subtilis foi a única espécie de bactéria relatada em 2 publicações produzindo valores de $4,9 \mathrm{U} \mathrm{mL}^{-1}$ por Suci et al. (2016), em resíduo de óleo de cozinha e 15,9 U mL $\mathrm{L}^{-1}$ por Aguiar et al. (2018), em resíduos de pesca.

$\mathrm{Na}$ literatura, os fungos filamentosos Rhizomucor meihei, Rhizopus oryzae, Pencillium camberti, Aspergillus niger, Penicillium simplicissimum, Trichoderma viride, Colletotrichum gloeosporioides, as leveduras Candida rugosa, Yarrowia lipolytica, Candida antarctica Geotrichium candidum e as bactérias Pseudomonas cepacia Pseudomonas aeruginosa, Pseudomonas fluorescens, Pseudomonas fragi, Bacillus thermocatenulatus, Staphylococcus hyicus, Staphylococcus aereus, Staphylococcus epidermidis, Bacillus strains e Bacillus subtilis são os mais reportados como bons produtores de lipase (Ramos-Sánchez et al. 2015; Messias et al. 2011; Kumar et al. 2011; Vakhlu 2006). De modo geral fungos filamentos e levedura são mais promissores para produção de lipase, entretanto como pode ser visto a exploração de espécie conhecidas se 
tornou corriqueira e repetitiva, faz-se assim a necessidade de busca e exploração de novos microrganismos selvagens que possam ser investigados quanto à produção de lipase utilizando os substratos mais promissores como resíduos de dendê, farelo de soja e arroz.

\subsection{Tempo de fermentação e indutores}

Um dos critérios para seleção dos trabalhos foi verificar o tempo de fermentação e a utilização ou não de substâncias indutoras ou para suplementação do meio.

Em relação ao tempo, as fermentações variaram em um período de 20 a 336 h, e o tempo de 48 h foi utilizado por 7 pesquisadores. O tempo de incubação é um fator importante para a produção de lipase extracelular, pois em períodos de incubação longos, a atividade da lipase diminuiu, o que pode ser devido ao esgotamento de nutrientes, acúmulo de produtos finais tóxicos e à mudança no $\mathrm{pH}$ do meio, ou perda de umidade (Sarat et al., 2010).

Vários pesquisadores relataram diferentes períodos de incubação para a produção ideal de lipase. A atividade máxima da lipase foi alcançada após 48 h de incubação por Penha et al. (2018), com A. niger. Rehman et al. (2019) relataram a atividade máxima da lipase por Pleurotus ostreatus IBL-02 após 72 h de incubação usando torta de óleo de canola como substrato e suplementando com glicose e ureia. Em outro estudo, a atividade máxima da lipase por Aspergillus niger ocorreu após 120 h de incubação (Putri et al., 2020). Moftah et al. (2012), obtiveram produção máxima de lipase por Candida utilisna após $120 \mathrm{~h}$ de incubação.

A obtenção das enzimas pode ocorrer principalmente via fermentação em estado sólido (FES) ou em fermentação submersa (FS). A maioria das pesquisas foram conduzidas em FES 73,3\% e 26,7\% foram experimentos realizados em fermentação submersa. A FES caracteriza-se pela ausência de água livre, porém, o substrato deve possuir umidade suficiente para suportar o crescimento e o metabolismo dos microrganismos, esse tipo de fermentação apresenta algumas vantagens como ambientes mais próximos do habitat natural, serve como suporte para crescimento das células microbianas disponibilidade, fonte alternativa com baixo valor comercial, características físicas e químicas que favorecem o crescimento de vários microrganismos, maior distribuição de oxigênio, menos problemas operacionais, equipamento e sistema de controle mais simples (Ramos-Sánchez et al., 2015; Ferreira et al., 2011; Pinto, et al. 2005), o que afirma a escolha dos autores selecionados por esse tipo de fermentação nos seus experimentos. Oliveira et al. (2013), reportam que os fungos têm maior aptidão em FES pela similaridade de seu ambiente natural, enquanto bactérias e leveduras, devido ao seu caráter unicelular ter maior habilidade em absorver nutrientes do meio.

Assim, observou-se que o processo de FES utilizando resíduos agroindustriais pode se tornar uma tecnologia vantajosa, pela conversão destes resíduos em produtos de alto valor agregado, como as enzimas (Ferreira et al., 2011).

Durante a fermentação alguns parâmetros ambientais são utilizados por diversos autores para otimização do processo como umidade, $\mathrm{pH}$, temperatura e atividade de água. Indutores do meio de cultivo é outro parâmetro bastante empregado para testar o aumento da eficiência dos microrganismos em produzir enzimas de interesse.

O azeite de oliva vem sendo a principal matéria-prima utilizada como indutora na produção de lipase, entretanto produzir lipases em escala industrial utilizando azeite de oliva como indutor sendo ele um produto de alto valor, pode ocasionar um aumento do valor do produto final, assim diversas pesquisas buscam suplementar os meios de cultivo com indutores mais baratos (Oliveira et al., 2013). A Figura 2 apresenta as publicações na qual se utilizou ou não indutores no meio de cultivo para potencializar a produção de lipase. 
Figura 2. Proporção das publicações em relação à utilização de indutores.

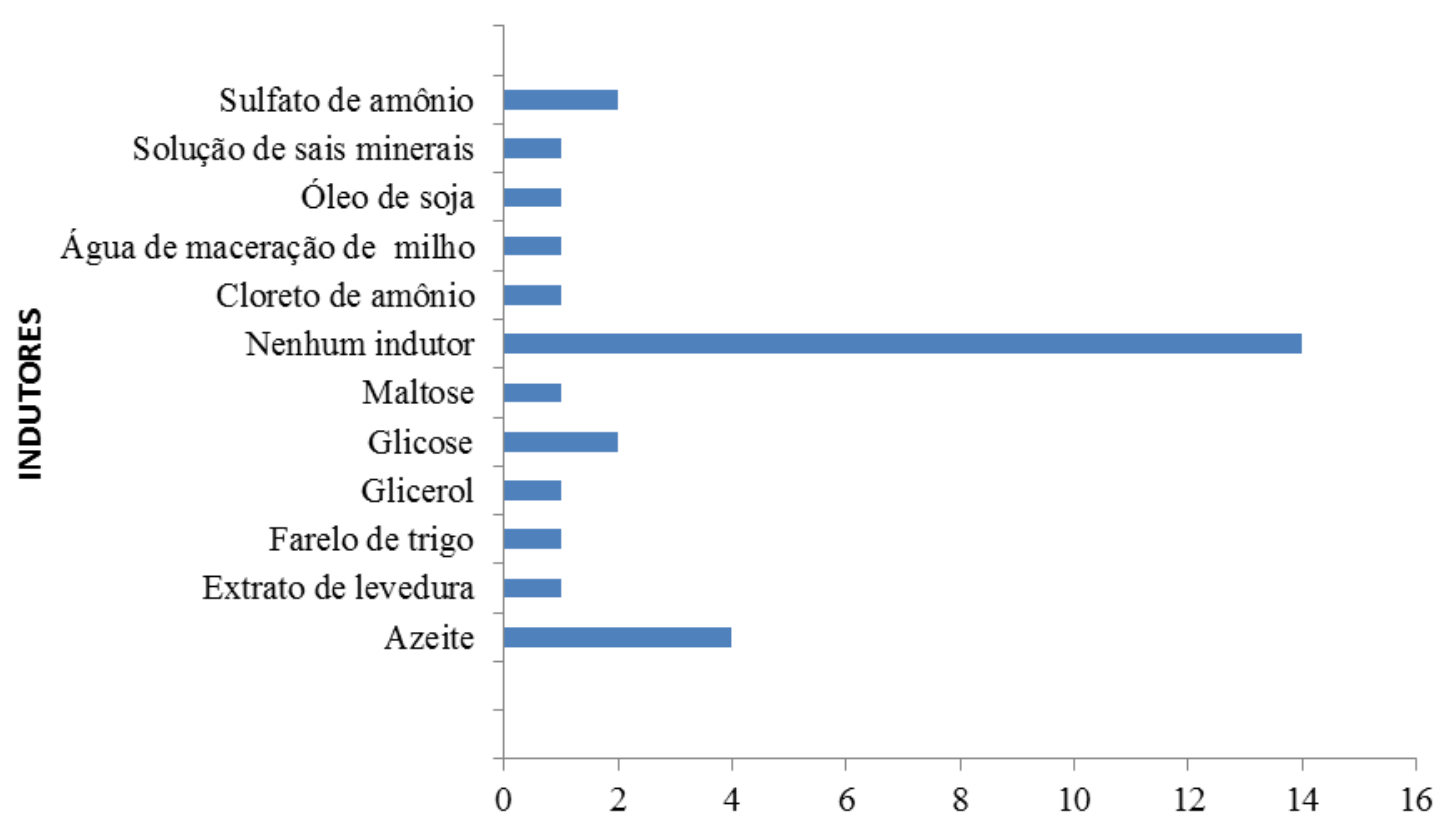

Fonte: Autores (2021).

Dentre as 30 publicações foi possível verificar que 14 (46,3\%) delas os autores não utilizaram nenhum tipo de indutor para suplementação do meio, ou seja, os resíduos agroindustriais por si só foram subtratos eficintes nutricionalmente para os microganismos, entretanto é possível verificar a utlização de azeite de óliva em quatro publicações para induzir a produção enzimática como também a utilização de fontes de nitrogênio como sulfato de amônio, extrato de levedura e cloreto de amónio, solução de sais.

$\mathrm{Na}$ maioria dos experimentos conduzidos pelos autores selecionados as máximas atividades lipolíticas foram encontrados quando se utilizou os indutores supracitados. Excluindo-se o azeite de oliva pelo seu auto valor, fontes de nitrogênio como uréia e sulfato de amônio torna-se um promissor indutor, diversos autores como Guedes et al. (2020), Penha et al. (2016) e Sarat et al. (2010) verificaram que tal composto contribui significadamente para produção de lipases por microrganismos, mostrando a importância da suplementação de uma fonte de nitrogênio ao meio. Penha et al. (2016), relata que após a adição do sulfato de amônio teve um aumento de 47,5\% a atividade lipolítica e Sarat et al. (2010), verificou os efeitos de diversos fontes de nitrogênio como ureia, peptona, extrato de malte, extrato de levedura, sulfato de amónio e $\mathrm{NH}_{4} \mathrm{H}_{2} \mathrm{PO}_{4}$ indicando que eles são significativos mas que, utilizando um planejamento experimental de Plackett-Burman, a ureia foi considerada altamente significativa para a produção de lipase. Pereira et al. (2019), teve seu melhor resultado na produção enzimática suplementando o meio com o extrato de levedura uma vez que é uma fonte de nitrogênio, bem como uma fonte de vitamina. Ramos-Sánchez et al. (2015), sugerem que a adição de fontes de nitrogênio orgânicos como ureia e hidrolisado de caseína tenha um aumento significativo na produção de lipase.

Em alguns casos, a adição de várias fontes de nitrogênio, carboidratos e indutores ao substrato não resultou em nenhum aumento significativo na síntese de lipase, evidenciando que, determinados substratos agroindustriais são suficientes para apoiar o crescimento celular e a produção de lipase em alto rendimento como pode ser observado nos trabalhos de Rehman et al., (2019) em torta de óleo de canola, Putri et al. (2020) com farelo de arroz. 


\section{Conclusão}

O Brasil detém uma forte economia voltada ao agronegócio, gerando inúmeros tipos de resíduos agroindustriais se destacando como principal gerador de publicações para a produção de lipase utilizando resíduos agroindustriais. Os resíduos oriundos da obtenção do azeite de dendê, farelo de arroz e soja foram os mais estudados como substrato.

O microrganismo mais utilizado para a produção de lipase foi o A. niger. Os trabalhos selecionados utilizaram em maior proporção a fermentação no estado sólido e a maioria das fermentações foram conduzidas no tempo de 48 h. Diversos resíduos por si só foram promissores para a produção de lipase entretanto a suplementação do meio com fontes lipídicas e principalmente de nitrogênio orgânico aumentaram significadamente a produção de lipase, utilizar resíduos agroindústrias e suplementar o meio com indutores com preços mais baixos, além de potencializar a produção pode tornar assim o produto final mais barato.

\section{Referências}

Amorim, G. M., dos Santos, T. C., Pacheco, C. S. v., Barreto, I. M. A., Freire, D. M. G., \& Franco, M. (2012). Fermentação de farelo de cacau por Aspergillus niger para obtenção de lipase. Estudos Tecnológicos Em Engenharia, 8, 24-27. https://doi.org/10.4013/ete.2012.81.04

Sarat Babu, I., Sita Kumari, K., \& Hanumantha Rao, G. (2010). Optimization of media constituents for the production of lipase in solid state fermentation by Yarrowia lipolytica from palm Kernal cake (Elaeis guineensis). Advances in Bioscience and Biotechnology, 2010.

Beltrán Ramos-Sánchez, L., Cujilema, M., Caridad Julián Ricardo, M., \& Cordova, J. (2015). Fungal Lipase Production by Solid-State Fermentation. Article in Journal of Bioprocessing \& Biotechniques. https://doi.org/10.4172/2155-9821.1000203.

Colla, L. M., Reinehr, C. O., \& Costa, J. A. v. (2012). Aplicações e produção de lipases microbianas. Revista CIATEC-UPF. 4(2). http://repositorio.furg.br/handle/1/4491.

Costa, T. M., Hermann, K. L., Garcia-Roman, M., de Valle, R. C. S. C., \& Tavares, L. B. B. (2017). Lipase production by aspergillus Niger grown in different agro-industrial wastes by solid-state fermentation. Brazilian Journal of Chemical Engineering, 34(2), 419-427. https://doi.org/10.1590/01046632.20170342s20150477.

Júnior M., W. G., Kamimura, E. S., Ribeiro, E. J., Pessela, B. C., Cardoso, V. L., \& de Resende, M. M. (2016). Optimization of the production and characterization of lipase from Candida rugosa and Geotrichum candidum in soybean molasses by submerged fermentation. Protein expression and purification, 123, 26-34.

Dobrev, G., Strinska, H., Hambarliiska, A., Zhekova, B., \& Dobreva, V. (2018). Optimization of Lipase Production in Solid-State Fermentation by Rhizopus Arrhizus in Nutrient Medium Containing Agroindustrial Wastes. The Open Biotechnology Journal, 12(1), 189-203. https://doi.org/10.2174/1874070701812010189.

dos Santos, P. S., Solidade, L. S., Souza, J. G. B., Sampaio, G., Braga Jr, A. C. R., de Assis, F. G. D. v., \& Leal, P. L. (2018). Fermentação em estado sólido em resíduos agroindustriais para a produção de enzimas: uma revisão sistemática. The Journal of Engineering and Exact Sciences, 4(2), 0181-0188. https://doi.org/10.18540/jcecvl4iss2pp0181-0188.

Eichler, P. (2018). Cultivo em estado sólido de aspergillus brasiliensis em bagaço de malte para a produção de lipases. In Dissertação (mestrado) Universidade Federal Do Rio Grande Do Sul Escola De Engenharia Departamento De Engenharia Química. https://lume.ufrgs.br/handle/10183/179533.

Farias, C. M., de Souza, O. C., Sousa, M. A., Cruz, R., Magalhães, O. M. C., de Medeiros, \& de Souza-Motta, C. M. (2015). African Journal of Biotechnology High-level lipase production by Aspergillus candidus URM 5611 under solid state fermentation (SSF) using waste from Siagrus coronata (Martius) Becari. African Journal of Biotechnology, 14(9), 820-828. https://doi.org/10.5897/AJB2014.14339.

Farias, M. A., Valoni, E. A., Castro, A. M., \& Coelho, M. A. Z. (2014). Lipase production by yarrowia lipolytica in solid state fermentation using different agro industrial residues. Chemical Engineering Transactions, 38, 301-306. https://doi.org/10.3303/CET1438051.

Ferraz, F. de S., Duarte, L., \& I. D.-2020 by A. E. (2020). Lipase extracelular do fungo Metarhizium anisopliae produzida a partir de residuos agroindustrais. In Avanços das pesquisas e inovações na engenharia química, 1, 99. https://doi.org/10.22533/at.ed.539202003.

Ferreira, O. E. (2011). Aproveitamento de subprodutos agroindustriais para a produção de amilases fúngicas: estudo de parâmetros fermentativos e caracterização das enzimas. In Dissertação (mestrado) - Universidade Estadual Paulista, Faculdade de Ciências Agrárias e Veterinárias, xv, 56 . Universidade Estadual Paulista (UNESP). https://repositorio.unesp.br/handle/11449/94886.

Garlapati, V. K., \& Banerjee, R. (2010). Optimization of lipase production using differential evolution. Biotechnology and Bioprocess Engineering, 15(2), 254-260. https://doi.org/10.1007/s12257-009-0163-3.

Godoy, M. G., Gutarra, M. L. E., Castro, A. M., MacHado, O. L. T., \& Freire, D. M. G. (2011). Adding value to a toxic residue from the biodiesel industry: Production of two distinct pool of lipases from Penicillium simplicissimum in castor bean waste. Journal of Industrial Microbiology and Biotechnology, 38(8), 945-953. https://doi.org/10.1007/s10295-010-0865-8. 
Guedes, E. H. S., Conte, C. G., de Abreu-Lima, T. L., \& Carreiro, S. C. (2020). Produção de lipase por leveduras isoladas de frutos de palmeiras. Research, Society and Development, 9(8), e394985487. https://doi.org/10.33448/rsd-v9i8.5487.

Gupta, R., Gupta, N., \& Rathi, P. (2004). Bacterial lipases: An overview of production, purification and biochemical properties. In Applied Microbiology and Biotechnology (Vol. 64, Issue 6, pp. 763-781). Springer. https://doi.org/10.1007/s00253-004-1568-8.

Imandi, S. B., Karanam, S. K., \& Garapati, H. R. (2010). Optimization of media constituents for the production of lipase in solid state fermentation by Yarrowia lipolytica from palm Kernal cake (Elaeis guineensis). Advances in Bioscience and Biotechnology, 01(02), 115-121. https://doi.org/10.4236/abb.2010.12016.

Jain, R., \& Naik, S. N. (2018). Adding value to the oil cake as a waste from oil processing industry: Production of lipase in solid state fermentation. Biocatalysis and Agricultural Biotechnology, 15, 181-184. https://doi.org/10.1016/j.bcab.2018.06.010.

Kumar, S., Katiyar, N., Ingle, P., \& Negi, S. (2011). Use of evolutionary operation (EVOP) factorial design technique to develop a bioprocess using grease waste as a substrate for lipase production. Bioresource Technology, 102(7), 4909-4912. https://doi.org/10.1016/j.biortech.2010.12.114.

Liu, X., \& Kokare, C. (2017). Microbial enzymes of use in industry. In Biotechnology of microbial enzymes (pp. 267-298). Academic Press.

Marques, A. G., Carvalho, S. Tamires., Soares, V. P. C., Monteiro, A. B. I., Guimarães, F. D. M., \& Franco, M. (2012). Fermentação de farelo de cacau por Aspergillus niger para obtenção de lipase. Estudos Tecnológicos Em Engenharia, 8(1), 24-27. https://doi.org/10.4013/ete.2012.81.04

Messias, J. M., Costa, B. Z. da, Lima, V. M. G. de, Giese, C., Dekker, R. F. H., \& Barbosa, A. de M. (2011). Lipases microbianas: Produção, propriedades e aplicações biotecnológicas. Semina: Ciências Exatas e Tecnológicas, 32(2), 213-234. https://doi.org/10.5433/1679-0375.2011v32n2p213

Moftah, O. A. S., Grbavčić, S., Žuža, M., Luković, N., Bezbradica, D., \& Knežević-Jugović, Z. (2012). Adding value to the oil cake as a waste from oil processing industry: Production of lipase and protease by Candida utilis in solid state fermentation. Applied Biochemistry and Biotechnology, 166(2), 348-364. https://doi.org/10.1007/s12010-011-9429-2

Nascimento, B. M. K., Martins, A. G. R., de Campos Takaki, G. M., da Silva, C. A. A., \& Okada, K. (2014). Utilização de resíduos agroindustriais para produção de tanase por Aspergillus sp isolado do solo da caatinga de Pernambuco, Brasil. E-Xacta, 1, 95-103. https://doi.org/http://dx.doi.org/10.18674/exacta.v7i1.1146

Oliveira, A. C. D., Vargas, J. V. C., Rodrigues, M. L. F., \& Mariano, A. B. (2013). Utilização de resíduos da agroindústria para a produção de enzimas lipolíticas por fermentação submersa. Revista Brasileira de Produtos Agroindustriais, 1, 19-26. http://www.deag.ufcg.edu.br/rbpa/rev151/Art1513.pdf

Oliveira, A. C., Amorim, G. M., Azevêdo, J. A. G., Godoy, M. G., \& Freire, D. M. (2018). Solid-state fermentation of co-products from palm oil processing: Production of lipase and xylanase and effects on chemical composition. Biocatalysis and Biotransformation, 36(5), 381-388. https://doi.org/10.1080/10242422.2018.1425400.

Oliveira, D. S., Ferraz, L. R., Treichel, H., \& Oliveira, D. (2014). Farelo de arroz como substrato para a produção de lipases microbianas. RAMVI, 1 . https://www.caxias.ideau.com.br/wp-content/files_mf/f2c455ba9f1b6a4ae1efc64cac85c950194_1.pdf

Aguiar, G. P. S. Martins, V. G. Martins, P. C. C. Boschero, R. A. \& Prenticehernández, C. (2018). Produção De Lipase Microbiana A Partir De Resíduos De Corvina. Revista de Engenharia e Tecnologia (Vol. 10, Issue 1). https://revistas.apps.uepg.br/index.php/ret/article/view/11980

Penha, E. das M., Viana, L. de A. N., Gottschalk, L. M. F., Terzi, S. da C., Souza, E. F. de, Freitas, S. C. de, Santos, J. de O., \& Salum, T. F. C. (2016). Aproveitamento de resíduos da agroindústria do óleo de dendê para a produção de lipase por aspergillus niger. Ciencia Rural, 46(4), 755-761. https://doi.org/10.1590/0103-8478cr20131673

Pereira, A. S., Shitsuka, D. M., Parreira, F. J., \& Shitsuka, R. (2018). Metodologia da pesquisa científica. UFSM. https://repositorio. ufsm. br/bitstream/handle/1/15824/Lic_Computacao_Metodologia-Pesquisa-Cientifica. pdf.

Pereira, A., Fontes-Sant'Ana, G. C., \& Amaral, P. F. F. (2019). Mango agro-industrial wastes for lipase production from Yarrowia lipolytica and the potential of the fermented solid as a biocatalyst. Food and Bioproducts Processing, 115, 68-77. https://doi.org/10.1016/j.fbp.2019.02.002

Pinto, G. A. S., de Brito, E. S., Andrade, A. M. R., Fraga, S. L. P., \& Teixeira, R. B. (2005). Fermentação em Estado Sólido: Uma Alternativa para o Aproveitamento e Valorização de Resíduos Agroindustriais Tropicais. Embrapa Agroindústria Tropical-Comunicado Técnico (INFOTECA-E). https://www.infoteca.cnptia.embrapa.br/bitstream/doc/426390/1/Ct102.pdf

Putri, D. N., Khootama, A., Perdani, M. S., Utami, T. S., \& Hermansyah, H. (2020). Optimization of Aspergillus niger lipase production by solid state fermentation of agro-industrial waste. Energy Reports, 6, 331-335. https://doi.org/10.1016/j.egyr.2019.08.064

Rajendran, A., \& Thangavelu, V. (2013). Utilizing agricultural wastes as substrates for lipase production by candida rugosa NCIM 3462 in solid-state fermentation: Response surface optimization of fermentation parameters. Waste and Biomass Valorization, 4(2), 347-357. https://doi.org/10.1007/s12649-012$9140-8$

Rehman, S., Bhatti, H. N., Bhatti, A., \& Asgher, M. (2011). Optimization of process parameters for enhanced production of lipase by Penicillium notatum using agricultural wastes. African Journal of Biotechnology, 10(84), 19580-19589. https://doi.org/10.5897/AJB11.1082

Rehman, S., Bhatti, H. N., Bilal, M., \& Asgher, M. (2019). Optimization of process variables for enhanced production of extracellular lipase by Pleurotus ostreatus IBL-02 in solid-state fermentation. Pakistan Journal of Pharmaceutical Sciences, 32(2), 617-624. https://europepmc.org/article/med/31081774

Rosa, M. F., Souza Filho, M. S. M., Figueiredo, M. C. B., Morais, J. P. S., Santaella, S. T., \& Leitão, R. C. (2011). Valorização de resíduos da agroindústria. https://www.researchgate.net/publication/259850615

Ruas, T. L., \& Pereira, L. (2014). Como construir indicadores de ciência, tecnologia e inovação usando Web of Science, Derwent World Patent Index, Bibexcel e Pajek. Perspectivas em Ciência da Informação, 19(3), 52-81. 
Research, Society and Development, v. 10, n. 2, e30710212537, 2021

(CC BY 4.0) | ISSN 2525-3409 | DOI: http://dx.doi.org/10.33448/rsd-v10i2.12537

Salihu, A., Alam, M. Z., AbdulKarim, M. I., \& Salleh, H. M. (2012). Lipase production: An insight in the utilization of renewable agricultural residues. In Resources, Conservation and Recycling (Vol. 58, pp. 36-44). Elsevier. https://doi.org/10.1016/j.resconrec.2011.10.007

Silveira, E. A., Tardioli, P. W., \& Farinas, C. S. (2016). Valorization of Palm Oil Industrial Waste as Feedstock for Lipase Production. Applied Biochemistry and Biotechnology, 179, 558-571. https://link.springer.com/article/10.1007/s12010-016-2013-z

Silveira, E., Tardioli, P. W., \& Farinas, C. S. (2015). Produção de lipase em cultivos sólidos e submersos utilizando resíduos do processamento do dendê e sua caracterização bioquímica. XX Simpósio Nacional De Bioprocessos XI Simpósio De Hidrólise Enzimática De Biomassa. https://doi.org/10.17648/sinaferm$2015-32122$

Souza, F. M., \& Aquino, L. C. L. (2013). Potencial da farinha de sementes de mangaba para a produção de lipase de Aspergillus niger: Influência da temperatura e umidade no processo. Scientia Plena, $8(12$ (b)).

Suci, M., Arbianti, R., \& Hermansyah, H. (2018). Lipase production from Bacillus subtilis with submerged fermentation using waste cooking oil. IOP Conference Series: Earth and Environmental Science, 105(1), 12126. https://doi.org/10.1088/1755-1315/105/1/012126

Takeyama, M., Kawaguti, H., Research, M. K.-, and, S., \& 2020, undefined. (2020). Resíduos agroindustriais como insumos promissores para obtenção de bioprodutos por leveduras-uma breve revisão. Rsdjournal. https://www.rsdjournal.org/index.php/rsd/article/view/4488

Thirunavukarasu, K., Purushothaman, S., Sridevi, J., Aarthy, M., Gowthaman, M. K., Nakajima-Kambe, T., \& Kamini, N. R. (2016). Degradation of poly(butylene succinate) and poly(butylene succinate-co-butylene adipate) by a lipase from yeast Cryptococcus sp. grown on agro-industrial residues. International Biodeterioration and Biodegradation, 110, 99-107. https://doi.org/10.1016/j.ibiod.2016.03.005

Tombini, J., Cunha, M. A. A. da, \& Lima, V. A. de. (2015). Fermentação Sólida de Farelo de Soja para Produção de Lipase: Avaliação dos Parâmetros Concentração de Inóculo, Umidade e Tempo de Cultivo. Anais Do V Simpósio de Bioquímica e Biotecnologia, 368-368. https://doi.org/10.5151/biochemvsimbbtec-22153

Treichel, H., de Oliveira, D., Mazutti, M. A., di Luccio, M., \& Oliveira, J. V. (2010). A review on microbial lipases production. In Food and Bioprocess Technology. 3(2), 182-196. Springer. https://doi.org/10.1007/s11947-009-0202-2

Vakhlu, J., \& Kour, A. (2006). Yeast lipases: Enzyme purification, biochemical properties and gene cloning. In Electronic Journal of Biotechnology. 9(1), 6985. https://doi.org/10.2225/vol9-issue1-fulltext-9 